\title{
NON-CANCELLATIVE POWER-FREE GROUPS
}

\author{
by R. G. WALKER \\ (Received 15 January, 1966)
}

1. Introduction. A. Geddes [1, Theorem 3.3] has shown that the partial algebraic system which he has called a power-free group need not be cancellative. In other words, there exist power-free groups containing at least one element $a$ with the property that $a b$ can equal $a c$ when $b \neq c$. In the present paper we propose to study the structure of such non-cancellative power-free groups, and we shall in fact obtain a complete solution to this problem.

2. Non-cancellators. We begin by restating some results obtained in [1]. In the following, $P$ denotes any power-free group.

An element $a$ of $P$ is regular in $P$ if the equation $a^{-1} x=a$ has at least one solution in $P$; otherwise $a$ is said to be irregular.

Lemma 2.1. If $a$ is an irregular element of $P$, then $a b=a c$ implies that $b=c$.

LeMma 2.2. If $a$ is a regular element of $P$, then $a b=a c$ implies that $b=c$ unless

$$
a b=a c=a^{-1},
$$

when it is possible to have $c=b^{-1}$ instead.

Both these results are immediate consequences of the main theorem concerning cancellation [1, Theorem 3.1].

We call an element $a$ of $P$ left-cancellative if, for all elements $b, c$ of $P$ for which $a b$ and $a c$ exist and are such that $a b=a c$, we have $b=c$; right-cancellative and cancellative are defined similarly. We recall that an element of a power-free group is right-cancellative if and only if it is left-cancellative. A non-cancellative power-free group is one which contains at least one non-cancellative element.

If $a$ is any non-cancellative element, then a non-cancellator of $a$ is any element $b \neq e$ for which $a b=a b^{-1}$. It is obvious that, if $b$ is a non-cancellator of $a$, then so also is $b^{-1}$. By Lemma 2.2, every non-cancellative element $a$ has at least one non-cancellator $b$, and we have $a b=a b^{-1}=a^{-1}$. If $x$ is any other non-cancellator of $a$, then, by Lemma 2.2, $a x=a^{-1}$. Thus $a x=a b$, and $x$ must be either $b$ or $b^{-1}$. We have thus shown that every non-cancellative element has exactly two distinct non-cancellators, and these are inverse to one another.

Lemma 2.3. If $a b=a b^{-1}$ with $b \neq e$, then (i) $b a=b^{-1} a=a^{-1}$ and (ii) $a^{-1} b=a^{-1} b^{-1}=a$.

Proof. (i) Since $b \neq e$, we must have $b \neq b^{-1}$, and hence, by Lemma 2.2, $a b=a b^{-1}=a^{-1}$. From $a b=a^{-1}$ we obtain $(a b) a=e$. Now, since $a b$ exists, so also does $b a$, and $b a \neq a$, since otherwise $b=e$. Hence $a(b a)=e$, showing that $b a=a^{-1}$. Similarly we may show that $b^{-1} a=a^{-1}$.

(ii) From part (i) we have $b a=b^{-1} a=a^{-1}$. By taking inverses we obtain

$$
(b a)^{-1}=\left(b^{-1} a\right)^{-1}=\left(a^{-1}\right)^{-1} \text {, }
$$

i.e. $a^{-1} b^{-1}=a^{-1} b=a$, as required. 
Lemma 2.3, (ii), shows that any non-cancellator of $a$ is also a non-cancellator of $a^{-1}$. Our next result shows that any non-cancellator must itself be cancellative.

Lemma 2.4. Suppose that $a$ is any non-cancellative element of $P$, and let $\alpha$ be any noncancellator of $a$; then $\alpha$ is cancellative.

Proof. Suppose that $\alpha$ is regular; then there is an $x$ in $P$ for which $\alpha^{-1} x=\alpha$. We first show that $x \neq a^{-1}$. Suppose, in fact, that $x=a^{-1}$; then, by the definition of $x$, we have $\alpha^{-1} a^{-1}=\alpha$, i.e. $(a \alpha)^{-1}=\alpha$. Now, since $\alpha$ is a non-cancellator of $a$, it follows that $a \alpha=a^{-1}$, and thus we obtain $\left(a^{-1}\right)^{-1}=\alpha$, i.e. $a=\alpha$. But $a \alpha$ is defined, since $\alpha$ is a non-cancellator of $a$, and hence $a=\alpha=e$ which is impossible, as $a$ is non-cancellative. We must therefore have $x \neq a^{-1}$, and thus $a^{-1} x$ is defined.

We have $a \alpha=a \alpha^{-1}=a^{-1}$, since $\alpha$ is a non-cancellator of $a$. Thus we obtain

$$
a^{-1} x=(a \alpha) x=\left(a \alpha^{-1}\right) x
$$

Now $\alpha^{-1} x$ is defined and equals $\alpha$, and $a \alpha$ is defined. It follows, by the partial associative law in $P$, that

$$
(a \alpha) x=a\left(\alpha^{-1} x\right)=a \alpha,
$$

giving $x=e$. But this implies that $\alpha^{-1} e=\alpha$, i.e. that $\alpha^{-1}=\alpha$, which gives $a=\alpha=e$, and this is impossible. Thus our original assumption that $\alpha$ is regular is false. Hence $\alpha$ is irregular, and, by Lemma 2.1 , it must be cancellative.

We have thus shown that any non-cancellative power-free group necessarily contains at least three cancellative elements, namely, the identity $e$ and two non-cancellators. The next result, which is the most crucial step in our argument, shows that in fact there must be exactly three cancellative elements in any non-cancellative power-free group.

THEOREM 2.5. Let $P$ be a non-cancellative power-free group. Then $P$ contains exactly two cancellative elements other than the identity (necessarily inverse to one another). Every other element of $P(\neq e)$ is non-cancellative and has as its two distinct non-cancellators these two cancellative elements.

Proof. Since $P$ is non-cancellative, there exists an element $a$ in $P$ such that $a$ is noncancellative. The element $a$ has two non-cancellators; let them be $\alpha$ and $\alpha^{-1}$.

By Lemma 2.3, (ii), $a^{-1}$ is also non-cancellative and its non-cancellators are also $\alpha$ and $\alpha^{-1}$. From Lemma 2.4, we know that $\alpha$ and $\alpha^{-1}$ are cancellative, and hence neither $a$ nor $a^{-1}$ can equal $\alpha$ or $\alpha^{-1}$. Thus, since none of these elements can equal the identity $e$ of $P$, it follows that $P$ contains at least five distinct elements, namely $e, a, a^{-1}, \alpha, \alpha^{-1}$. If $P$ contains exactly these five elements, the proof is finished. We therefore assume that $P$ contains more than five elements.

Let $c$ be any element of $P$ distinct from $e, a, a^{-1}, \alpha, \alpha^{-1}$. We shall show that $c$ is noncancellative and has $\alpha$ and $\alpha^{-1}$ as its non-cancellators, thereby completing the proof of the theorem.

Since $c \neq a^{-1}$, the product $c a^{-1}$ exists; let us denote it by $x$. Suppose that $x=a$; then $c a^{-1}=a$, so that we have, taking inverses, $a c^{-1}=a^{-1}$. But $a^{-1}=a \alpha$, since $\alpha$ is a non-

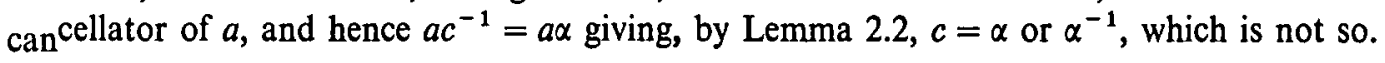


Thus we must have $x \neq a$, and so $x a$ exists; further it equals $\left(c a^{-1}\right) a$, i.e. $c$.

Now, since $c \neq \alpha$, it follows that $c \alpha$ exists, and thus we have

$$
c \alpha=(x a) \alpha=x(a \alpha)
$$

by the partial associative law in $P$, since $a \alpha$ exists and equals $a^{-1}$, and also $x \neq a^{-1}$, since otherwise we should have $a^{-1}=c a^{-1}$ giving $c=e$, which is not so. Further $a \alpha=a \alpha^{-1}$, since $\alpha$ is a non-cancellator of $a$, and thus, from the above, we obtain

$$
c \alpha=x\left(a \alpha^{-1}\right)=(x a) \alpha^{-1}=c \alpha^{-1},
$$

our use of the partial associative law being justified by the fact that $x \neq a$ and $x a=c \neq \alpha^{-1}$.

Accordingly we have shown that $c \alpha=c \alpha^{-1}$; in other words, that $c$ is non-cancellative with $\alpha$ and $\alpha^{-1}$ as its non-cancellators, as required.

3. The structure theorem. In [2] a complete account of cancellative power-free groups is given. The following lemma shows that, if we remove the two non-cancellators from a noncancellative power-free group $P$, we obtain a cancellative power-free group $Q$. We are then able to apply results from the cancellative theory to $Q$.

Lemma 3.1. Let $P$ be a non-cancellative power-free group with non-cancellators $\alpha$ and $\alpha^{-1}$, and let $Q$ be the set consisting of all the elements of $P$ with the exception of $\alpha$ and $\alpha^{-1}$. Then, if $x$ and $y$ are distinct elements of $Q$, their product $x y$ is also in $Q$, and, in fact, under the multiplication induced by the multiplication in $P$, the set $Q$ is a cancellative power-free group.

Proof. Let $x$ and $y$ be distinct elements of $Q$, and suppose that $x y$ is not in $Q$; then $x y=\alpha$ or $\alpha^{-1}$. Now $x \neq e$, since $y$ is in $Q$ and hence is not equal to $\alpha$ or $\alpha^{-1}$. Thus, as $x$ is in $Q$, we have $x \neq e, \alpha, \alpha^{-1}$, and, accordingly,

$$
x^{-1}(x y)=x^{-1} \alpha \text { or } x^{-1} \alpha^{-1}=x,
$$

by Theorem 2.5. This, however, implies that $y=x$, which is a contradiction, and consequently $x y$ must be in $Q$.

It follows that $Q$ is closed under the multiplication induced by $P$; further, the partial associative law holds in $Q$, since it holds in $P$, and also $Q$ has an identity, namely the identity $e$ of $P$. Finally, if $x$ is any element of $Q$, then its inverse $x^{-1}$ in $P$ is also in $Q$, and so every element of $Q$ has an inverse. We conclude that $Q$ is a power-free group.

Suppose that $Q$ is non-cancellative; then $Q$ contains two non-cancellators. It is clear that these must also be non-cancellators of $P$; but this is impossible, since $P$ has only two noncancellators and these are not members of $Q$. Accordingly $Q$ is cancellative.

We note that, if $Q$ is the cancellative power-free group derived from a non-cancellative power-free group $P$ by removing its non-cancellators, then $P$ is the only non-cancellative power-free group from which $Q$ can be derived by this process. In fact, the multiplication of $P$ is completely determined by that of $Q$, since, if $\alpha$ and $\alpha^{\prime}$ are to be the two additional elements of $P$, we must have $\alpha \alpha^{\prime}=\alpha^{\prime} \alpha=e$ and $\alpha x=x \alpha=\alpha^{\prime} x=x \alpha^{\prime}=x^{-1}$ for all $x \neq e$ in $Q$. 
LEMMA 3.2. Let $P$ be a non-cancellative power-free group, and $Q$ the cancellative power-free group obtained from $P$ by removing its non-cancellators $\alpha$ and $\alpha^{-1}$; then all the elements of $Q$, other than $e$, are irregular in $Q$, and, further, for every pair of irregular elements $x$ and $y$ of $Q$ with $x \neq y$, we have $x(x y)=y^{-1}$.

Proof. Suppose that $Q$ contains a regular element $a \neq e$; then there is an element $b$ of $Q$ such that $a^{-1} b=a$ in $Q$, and hence in $P$. However, in $P$, since $a \neq e, \alpha, \alpha^{-1}$, we have, by Theorem 2.5, that $a^{-1} \alpha=a$. Thus $a^{-1} b=a^{-1} \alpha$, and so, by Lemma $2.2, b=\alpha$ or $\alpha^{-1}$, which is impossible, since $b$ is in $Q$. It follows that all the elements of $Q$, other than $e$, are irregular.

Let $x$ and $y$ be any two irregular elements of $Q$; then, by [2, Lemma 2.1], we must have either $x(x y)=y^{-1}$ or $x(x y)=x^{-1} y$ in $Q$. Suppose that $x(x y)=x^{-1} y$; then this will also hold in $P$. However, in $P$, we have $x^{-1}=\alpha x$, and, accordingly, $x(x y)=(\alpha x) y$. Now, by Lemma 3.1, $x y$ is in $Q$, since both $x$ and $y$ are in $Q$, and thus $\alpha \neq x y$, i.e. $\alpha(x y)$ is defined. We therefore obtain $x(x y)=\alpha(x y)$, and Lemma 2.2 then implies that $x=\alpha$ or $\alpha^{-1}$, which contradicts the fact that $x$ is in $Q$. From this it follows that $x(x y) \neq x^{-1} y$, and so we have the required result.

LEMMA 3.3. If $Q$ is the power-free group derived from a non-cancellative power-free group by removing its non-cancellators, then $Q$ is either the power-free group of order 3 or the quaternion power-free group.

Proof. If $Q$ is not the power-free group of order 3, then the order of $Q$ must be greater than 3. By Lemma 3.2, we know that, apart from the identity, $Q$ consists entirely of irregular elements satisfying $x(x y)=y^{-1}$ for $x \neq y$. We now apply [2, Lemma 3.3], which states that the only cancellative power-free group with these properties is the quaternion power-free group, to obtain the result.

The last lemma shows that there are at most two non-cancellative power-free groups, one corresponding to each $Q$. We now take the two possible cancellative power-free groups $Q$, and consider the result of adding to them two non-cancellators $\alpha$ and $\alpha^{-1}$. In the case when $Q$ is the power-free group of order 3 , we obtain a system with the following multiplication table:

\begin{tabular}{l|lll|ll}
$e$ & $a$ & $a^{-1}$ & $\alpha$ & $\alpha^{-1}$ \\
\hline$e$ & $e$ & $a$ & $a^{-1}$ & $\alpha$ & $\alpha^{-1}$ \\
$a$ & $a$ & $*$ & $e$ & $a^{-1}$ & $a^{-1}$ \\
$a^{-1}$ & $a^{-1}$ & $e$ & $*$ & $a$ & $a$ \\
$\alpha$ & $\alpha$ & $a^{-1}$ & $a$ & $*$ & $e$ \\
$\alpha^{-1}$ & $\alpha^{-1}$ & $a^{-1}$ & $a$ & $e$ & $*$
\end{tabular}

This is in fact the non-cancellative power-free group of order 5 considered in [1, pp. 397-398]. 
Corresponding to the quaternion power-free group, we obtain the following system of order 9:

\begin{tabular}{l|lllllll|ll}
$e$ & $a$ & $a^{-1}$ & $b$ & $b^{-1}$ & $c$ & $c^{-1}$ & $\alpha$ & $\alpha^{-1}$ \\
\hline$e$ & $e$ & $a$ & $a^{-1}$ & $b$ & $b^{-1}$ & $c$ & $c^{-1}$ & $\alpha$ & $\alpha^{-1}$ \\
$a^{-1}$ & $*$ & $e$ & $c$ & $c^{-1}$ & $b^{-1}$ & $b$ & $a^{-1}$ & $a^{-1}$ \\
$b$ & $a^{-1}$ & $e$ & $*$ & $c^{-1}$ & $c$ & $b$ & $b^{-1}$ & $a$ & $a$ \\
$b^{-1}$ & $c^{-1}$ & $c$ & $*$ & $e$ & $a$ & $a^{-1}$ & $b^{-1}$ & $b^{-1}$ \\
$c$ & $b^{-1}$ & $c$ & $c^{-1}$ & $e$ & $*$ & $a^{-1}$ & $a$ & $b$ & $b$ \\
$c^{-1}$ & $c$ & $b$ & $b^{-1}$ & $a^{-1}$ & $a$ & $*$ & $e$ & $c^{-1}$ & $c^{-1}$ \\
\hline$\alpha$ & $c^{-1}$ & $b^{-1}$ & $b$ & $a$ & $a^{-1}$ & $e$ & $*$ & $c$ & $c$ \\
$\alpha^{-1}$ & $\alpha$ & $a^{-1}$ & $a$ & $b^{-1}$ & $b$ & $c^{-1}$ & $c$ & $*$ & $e$ \\
$\alpha^{-1}$ & $a^{-1}$ & $a$ & $b^{-1}$ & $b$ & $c^{-1}$ & $c$ & $e$ & $*$
\end{tabular}

With the exception of the partial associativity condition, the axioms of a power-free group are obviously satisfied by this system. It has been checked that the partial associative law does in fact hold, its verification being greatly facilitated by the fact that it is known to hold in the quaternion power-free group.

Thus both the cancellative power-free groups $Q$ appearing in Lemma 3.3 do indeed correspond to non-cancellative power-free groups. Accordingly, we can state

THEOREM 3.4. There are exactly two non-cancellative power-free groups, one being of order 5 , the other of order 9.

This result completes our study of the structure of non-cancellative power-free groups. Combining the results of [2] with those of the present paper, we can classify all power-free groups as follows:

(i) Those which can be derived from groups containing no elements of order 2 by the omission of squares; these are the complete power-free groups, and are, of course, cancellative.

(ii) The quaternion power-free group; this is the only incomplete cancellative power-free group.

(iii) The two non-cancellative power-free groups. 
I am grateful to Dr A. Geddes for a number of suggestions which have led to an improvement in the presentation of this paper.

\section{REFERENCES}

1. A. Geddes, Power-free groups, Proc. Cambridge Philos. Soc. 60 (1964), 393-408.

2. A. Geddes and R. G. Walker, The structure of cancellative power-free groups, Proc. Glasgow Math. Assoc. 7 (1966), 199-206.

UNIVERSITY OF GLASGOW

GlasGow, W.2 